stopped the stirrer. In some of the first experiments, the treated starch was washed with cold water, and the behavior of the resulting solution noted in the table. The opalescence and precipitates with alcohol indicate that the reaction does not proceed in two definite, separate steps, but that dextrin begins to be formed in small quantities as soon as the soluble starch formation begins. Undoubtedly all products designated as "Cold Water Paste" contained a good deal of dextrin.

It would be comparatively easy to remove or neutralize the small amount of acid required if the conversion to dextrin were made at $100^{\circ}$ or over, and it would appear that this would be an easy way of making a good white dextrin. If the starch were dried somewhat before use, less acid would probably be required and the product would not lump, hence would not need grinding. The method does not seem very well adapted to producing soluble starch, and it is evident that the temperatures given for this by Browning and Barlow require the use of larger quantities of acid than would be economically practicable, unless the time of treatment were much increased.

Schoot OF CheMrstry

UNIVERSTY" OF MiNNESOTA, MinNeapolis

\section{THE DIRECT AND THE INVERT POLARIZATION OF PURE SUCROSE ${ }^{1}$}

By HERBERT S. WALEFR

Considerable doubt has arisen in recent years as to the correctness of our present polarimetric methods of determining sucrose. At the last International Congress of Applied Chemistry a paper was presented by Bates and Jackson, indicating that the Ioo point of the saccharimeter is approximately $0 . I^{\circ}$ too high. This subject was deemed of sufficient importance to warrant appointing a special committee for its investigation, to report at the next meeting of the Congress.

At the same session, on motion of Prof. Herzfeld, a committee was also appointed to re-determine the correctness of the factor 142.66 now used in the ClergetHerzfeld method for the determination of sucrose by double polarization.

Since then, investigations by Steurwald, in Java, and Stanek, in Bohemia, tend to prove that the Clerget factor 142.66 is from 0.2 to 0.3 too low, Stanek claiming, however, that if the invert polarization be made within 5 minutes after completing the volume of the inverted solution, the original Herzfeld factor holds good, but owing to a slight muta-rotation of the invert sugar, a constant invert polarization is not reached until I 5 to 20 minutes after completing to volume.

Both these investigators apparently find, or assume, the direct polarization of pure sucrose to be 100.0 .

Since it will probably be several years before reports from the committee officially appointed to investigate these two subjects may be expected, the following experimental data may be of some interest:

An attempt was made to prepare pure sucrose by the customary method of precipitation from a hot,

'Paper presented at the Annual Meeting of the Hawaiian Chemists' Association, Oetober 22, 1914. saturated solution by absolute alcohol, starting with the best grade of "domino" sugar. The sugar thus obtained was re-dissolved, re-precipitated, washed on a suction filter with alcohol, and finally air-dried

\section{DETAILS OF EXPERIMENTS}

SACCHARIMETER-S. \& H. No. 8800 , double field.

LIGHT-IOO W, concentrated filament tungsten.

IIGHT FILTER-3 $-3 \mathrm{~cm}$. of 3 per cent potassium bichromate solution.

IOO POINT OF INSTRLMEN verified to $0.0 \mathrm{I}^{\circ}$ by comparison with a quartz plate standardized by the Bureau of Standards.

TUBE LENGTH-measured by a standardized comparator to $0.03 \mathrm{~mm}$.

FLASK used for making up solutions for direct polarization and for inversion made with special narrow neck-calibrated to $0.02 \mathrm{cc}$.

CONCENTRATION OF SOLUTIONS FOR DIRECT POLARIZATIONdetermined by weight as well as by volume, then calculated to grams solids per roo cc., thus eliminating correction for moisture in the sugar and giving concentration exact to o.or $\mathrm{g}$. per IOO cc., or $0.004^{\circ} \mathrm{V}$.

VOLUME OF SOLUTION LSED FOR INVERSION-checked by weight. After inversion and completion to volume, cooled solution was placed in the same "inversion tube" used for direct polarization and allowed to stand for approximately half an hour in the saccharimeter trough till it had assumed the same temperature as the instrument and the atmosphere, before reading.

WEIGHTS USED-corrected to $0.000 \mathrm{I}$. by standard weights.

THERMOMETERS-corrected by gas thermometer.

"PROBABLE FRROR" OF READING INSTRUMENT (average of IO readings)-For direct polarization $0.00^{\circ} \mathrm{V}$. For invert polarization $0.02^{\circ} \mathrm{V}$.

for several days. It was then found to contain: Moisture, 0.02 per cent; ash, 0.004 per cent; reducing sugars, less than 0.016 per cent (using Ost's solution).

The direct polarization of this sugar, using 26.000 g. dry sugar (weighed in air with brass weights) in 100 true cc. was, at $22.0^{\circ} \mathrm{C}$, after adding 0.06 for temperature correction, 99.86. Adding 0.02 as a correction for reducing sugars and ash would give 99.88 as the polarization of $26 \mathrm{~g}$. pure sucrose dissolved in 100 true $c c$. solution at $20^{\circ} \mathrm{C}$.

Fifty cc. of the solution used for direct polarization were then inverted by the Herzfeld method and eventually polarized at $22.2^{\circ} \mathrm{C}$. Using the factor I 42.66 , the calculated per cent sucrose of this sugar, corrected for reducing sugars and ash, was 100.07 .

As a check on the above determination, a sample of pure sucrose was obtained from the Bureau of Standards, Washington, D. C. Its certified analysis was as follows: Moisture, 0.002 per cent.; ash, 0.002 per cent; invert sugar, less than 0.003 per cent. Its direct polarization, then, should exceed $99.99^{\circ}$.

At $23.95^{\circ} \mathrm{C}$, the direct polarization actually found was, corrected for temperature, 99.90. Its sucrose by the Clerget-Herzfeld method, using the factor I42.66, was 100.09 , showing a difference between direct polarization and "sucrose by Clerget" of $0 . x 9$ per cent.

From the last experiment it appears that the Clerget factor, if designed to give a reading of roo per cent 
for pure sucrose with the present sugar scale, should be increased from 142.66 to 142.78 , while if it is intended to give the same figure as the direct polarization of pure sucrose, regardless of the scale used, which is more logical, it should be further increased to 142.92 .

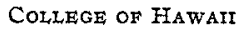

\section{THE NITROGEN AND FAT IN SHORT STAPLE COTTON-} SEED

By C. A. Wells AND F. H. Smith

Received December 21, 1914

The cotton plant breeders of the past have directed their efforts mainly towards increasing the yield and length of lint of cotton as well as its power of resistance, while the question of next importance, $i$. e., the relative percentage of nitrogen and fat in different types of seed, has received little consideration. The manufacture and preparation of cottonseed oil and its products and of cottonseed meal constitute important industries within themselves. Modern and intelligent management of these industries is beginning to demand a more thorough understanding of the properties of the different varieties of seed. Already there is a wide-spread and growing endeavor on the part of the manufacturer to purchase seed containing a high percentage of fat and nitrogen, particularly the latter.

A study has been made in this laboratory of the commercially important chemical constituents of eighteen varieties of short staple cottonseed. These varieties were selected as typical for the upland sections of Alabama, Georgia and other states similarly situated. They were grown upon the same kind of soil (a red clay) which had been uniformly fertilized. This was deemed necessary because experience had indicated that both the type of soil and nature of fertilizer may affect the nitrogen and oil content of the seed. The cotton was ginned in one gin under conditions as nearly uniform as possible and the remaining lint was removed by hand with a scalpel. Even though tedious, this was essential because, as will be shown in Table I, the lint not removed from the seed by the gin may vary from 97 to 214 lbs. per I000 $\mathrm{lbs}$. of seed, so that it is inaccurate to base analytical results upon the original weight of seed, as has often been done, without proper consideration for the unremoved lint. The,hulls were removed by hand and the kernels analyzed, using the Gunning-ArnoldDyer modification of the Kjeldahl method for nitrogen, allowing for nitrates, and the vacuum-sulfuric acid procedure for moisture. It was not found feasible to determine the moisture in kernels or in cottonseed meal by the usual method of drying in an oven, because decomposition took place even at $60^{\circ}$. Similarly it was found necessary to dry the flasks containing the ether extracts by immersing them deeply into the boiling water of the water bath instead of drying on the water bath or in the drying oven.

The analytical results are given in Table $I$ in order of nitrogen-ascendency in two groups of nine varieties each.

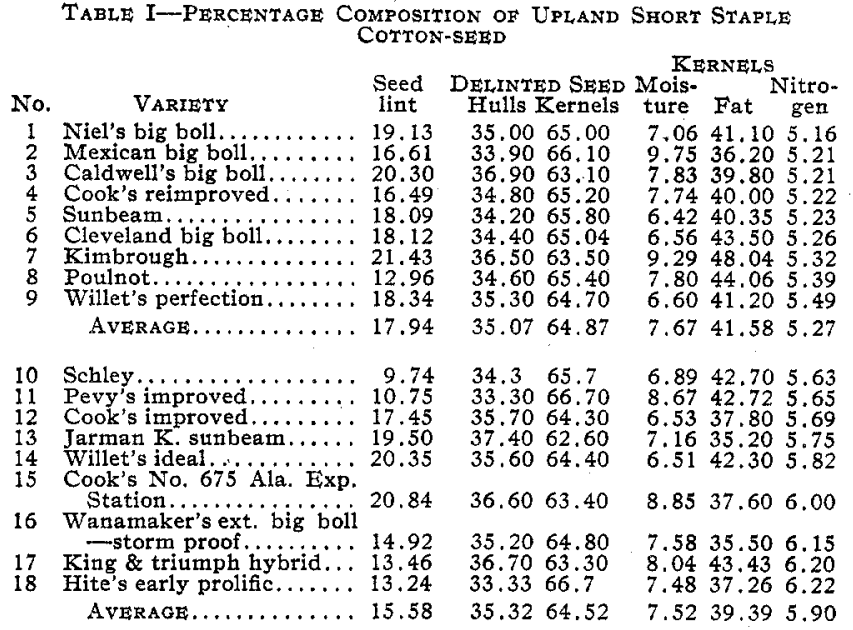

Attention has been called already to the difference in lint which the process of ginning may leave on the seed. From Table I it will be seen, also, that the fat in the kernels may vary from 35.2 to 48.04 per cent and the nitrogen from 5.I6 to 6.22 per cent. If the average be taken for the two arbitrarily chosen groups of nine varieties each as given in the table, it will be seen that the fat varies only slightly, especially if consideration is had for the difference in lint on the seed, while the nitrogen shows a variation of approximately io per cent. Thus a ton of mixed cottonseed from the second group would contain 8.3 lbs. more nitrogen and $13.6 \mathrm{lbs}$. less oil than a ton of seed made up similarly from Group I. This amount of nitrogen has a value of approximately $\$ 2.00$ and the oil a value of about 50 cents for the expressible portion with a slight feeding value for the non-expressible portion, leaving a balance of approximately $\$ I .50$ a ton in favor of the high nitrogen seed. This means a great saving on the total of $5,000,000$ tons of cottonseed crushed annually in the South. It will be interesting in the future to ascertain if the variations noted here are constant or if they change with the seasons.

I,ABoratory OF Chemistry, Georgia Experiment STATION EXPERTMENT, GHORGIA

\section{UNSAPONIFIABLE MATTER IN GREASES}

By E. TwITCHELL

Received November 30, 1914

The following method of determining unsaponifiable matter in greases has been in use under my direction in the laboratory of the Emery Candle Co. for five or six years.

Five grams of the sample (or preferably of the fatty acid prepared for "titer test," as this is cleaner) are saponified with alcoholic potash in a dish and evaporated nearly to dryness. A little aicohol is added and then water, and the solution obtained is washed into a separatory funnel. The ratio of alcohol to water in this soap solution should be about I : 4 , and the total volume of the soap solution $5_{50}$ to $200 \mathrm{cc}$. The soap solution is shaken twice with ether, using about so cc. each time. The ether extracts are united, washed once with water, then shaken 